\title{
Quadrotor with Image Processing Capabilities
}

\author{
Suraj Bobade ${ }^{1}$, Rohit Bhide ${ }^{2}$, Shreyash Durge ${ }^{3}$, Shrinivas Chavan ${ }^{4}$ \\ 1,2,3,4 BTech Electrical, VJTI, Mumbai.
}

\begin{abstract}
Quadrotor helicopters or simply called as Quadrotors represent a useful class of flying robots and are an emerging popular platform for unmanned aerial vehicle $(U A V)$ research and have applications of their own. Due to their simplicity of construction, easy maintenance, their ability to hover effortlessly, strong abilities of VTOL, vertical take off and landing (VTOL) capabilities, high manoeuvrability and controllability (especially in indoor areas). In this paper, we present the development of an unmanned UAV, called Quadrotor, which has the abilities to perform acrobatic movements like flips, rolls, barrels etc. It is also able to capture real time videos using a RF wireless camera. These videos are converted to snapshots which are then processed using MATLAB-Image Processing Toolbox to count the number of persons in that specific captured image.
\end{abstract}

Keywords: Quadrotor, UAV, Image Processing

\section{Introduction}

Quadrotor helicopters, simply called as Quadrotors, are robust and simple helicopters which do not have the complicated swash plates and linkages found in conventional rotorcraft. These are an emerging rotorcraft concept for unmanned aerial vehicle (UAV) platforms. The vehicle consists of four rotors in total located at the four corners of the aircraft, with two opposite pairs of clockwise rotating $(\mathrm{CW})$, fixed-pitch or variable pitch, blades and the another two opposite pairs of counter clockwise rotating $(\mathrm{CCW})$ blades. This configuration also offers the advantage of lateral motion without changing the pitch of the propeller blades. An example of such a quadrotor is shown in Figure 1.1. These are powered by Ni-Cd or Li-Poly cells and use rate feedback from MEMS gyroscopes.

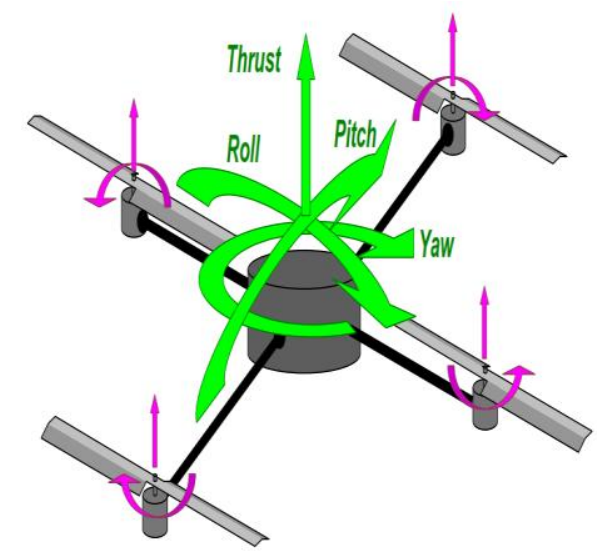

Figure. 1.1 Aerodynamic of Quadrotor

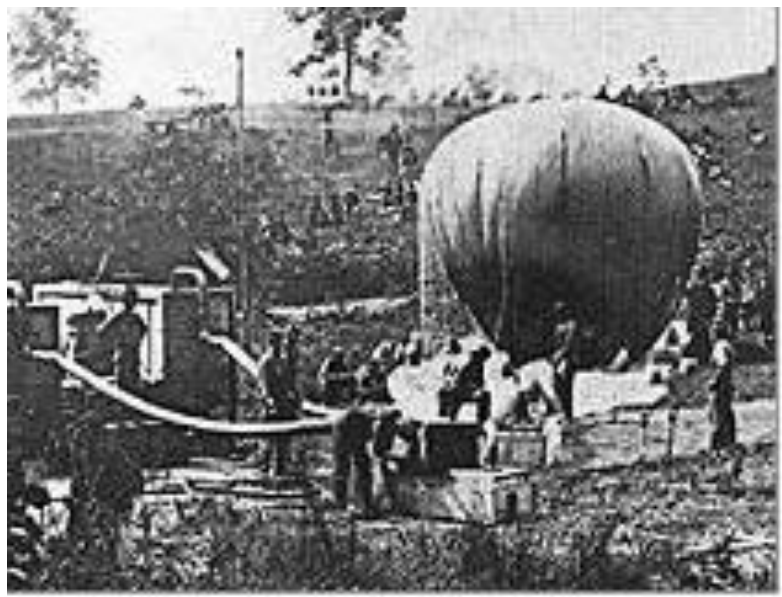

Figure 2 
1.1 History

Quadrotor was first experimented in 1907 by Breguet and Richet [1]. Their large, heavy but manned vehicle lifted only over a small height and for short duration. Since then, there has been tremendous research and development in the field of rotorcraft, but the Quadrotor concept did not get much attention until the last few years. Interestingly, the first recorded construction of a quadrotor helicopter was in 1922 when Georges de Bothezat and Ivan Jerome built and flew the "Flying Octopus" [2]. Also Pre-aviation UAVs, such as Perley's aerial bomber, shown in Figure 2, were generally nothing more than floating payloads with timing mechanisms designed to drop explosives in enemy territory. Later during the 1960s, surveillance drones were used for aerial reconnaissance in Vietnam. Lately after Operation Desert Storm, UAV development boomed in the United States. Current market studies estimate that worldwide UAV spending will become double during the next 10 years, from $\$ 4.9$ billion to $\$ 11.5$ billion annually. This will make the UAV market reach a colossal amount of $\$ 80$ Billion over the next decade [3]

\subsection{Flight Control Mechanism}

Quadrotor employs four rotors to create differential thrust, due to this the craft is able to hover and move without the complex system of linkages and blade elements present on standard single rotor conventional vehicles such as helicopters. Here only four actuators (rotors) are used to control all six degrees of freedom (DOF). The four actuators directly impact z-axis translation (altitude) and rotation about each of the three principal axes. The other two DOF are translation along the $\mathrm{x}$ - and $\mathrm{y}$-axis. These two remaining DOF are coupled, meaning they depend directly on the overall orientation of the vehicle (the other four DOF).Control of a Quadrotor is achieved by controlling different speeds to different propellers, which in turn produces differential aerodynamic forces and consequently the desired moments. These are as follows:

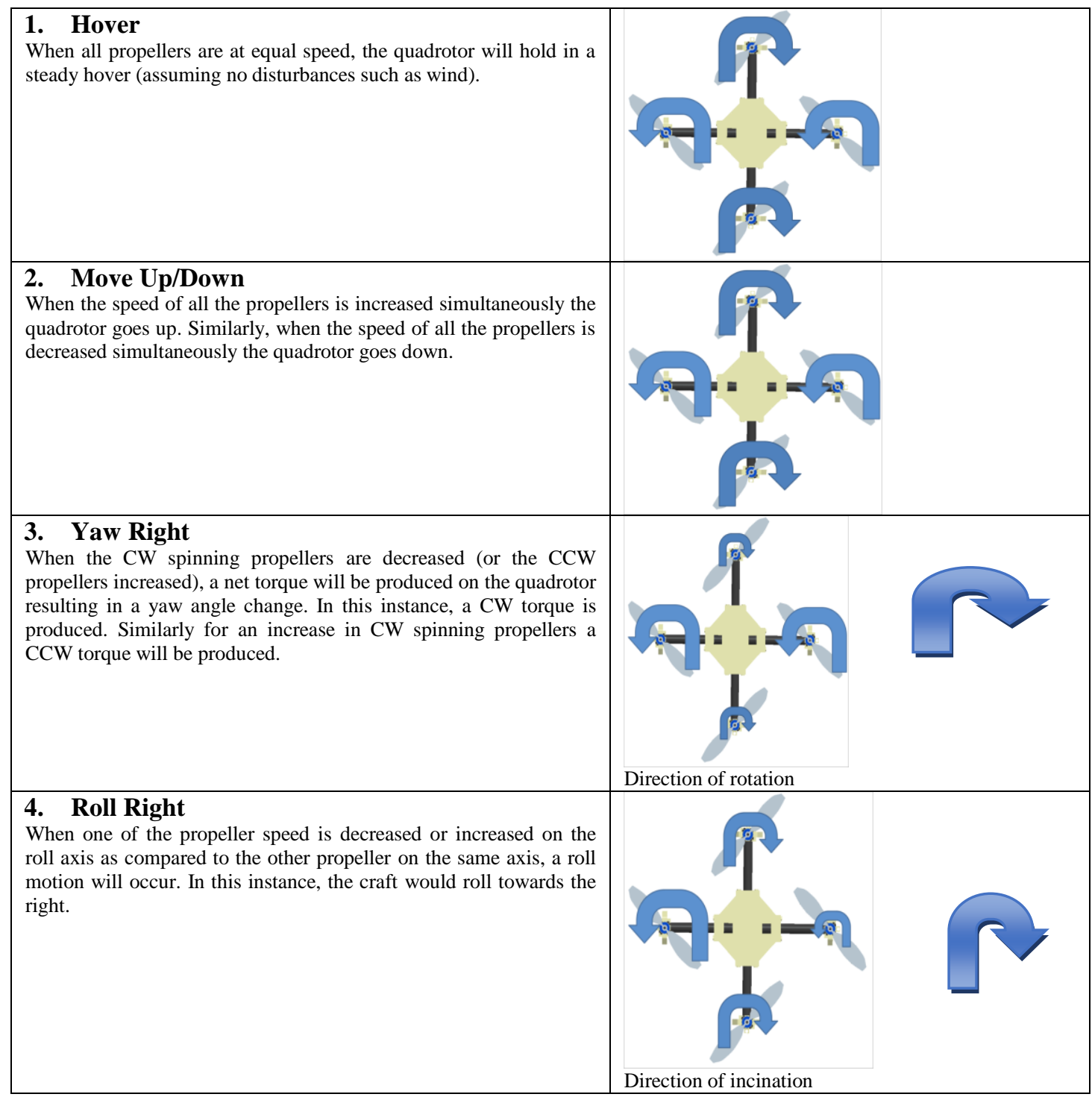




5. Pitch Up
Similar to the roll axis, if either propeller is changed on the pitch
axis, the axis will rotate in the direction of the smaller thrust. In this
instance, the craft nose would pitch up towards the reader (out of the
page) due to the differential on the pitch axis.
Direction of inclination

\subsection{Dynamics Of Quadrotor}

\section{Modelling of The Quadrotor}

The dynamic model of the quadrotor is derived under the following assumptions:

1. Quadrotor is a rigid body and perfectly symmetrical.

2. The centre of mass of the quadrotor is at the intersection of the cross frame.

3. The propellers have a fixed pitch.

4. The hub forces, horizontal forces on the blades, and friction/drag induced by the wind on the blades will be ignored in the $\mathrm{x}$ - and $\mathrm{y}$ - directions.

5. The earth fixed axis system will be regarded as an inertial reference frame.

For a Quadrotor, its attitude about all 3 axis of rotation is known with 6 states called the Euler angles (Roll - Pitch - Yaw) and the angular velocities [P Q R]. Yet another 6 states are necessary which are the position of the centre of gravity (CG) [X Y Z] and the respective linear velocity components [U V W] relative to the fixed frame. Hence we can say that the Quadrotor has 12 states that describe 6 degrees of freedom. The orientation of the mobile frame relative to the fixed one can be found using a Rotation Matrix (S), which is the product between three matrices R' $(\Theta), R^{\prime}(\phi)$ and R' $(\psi)$.

$$
\begin{gathered}
\mathbf{R}^{\prime}(\phi)=\left[\begin{array}{ccc}
1 & 0 & 0 \\
0 & \cos \phi & \sin \phi \\
0 & -\sin \phi & \cos \phi
\end{array}\right] \mathbf{R}^{\prime}(\theta)=\left[\begin{array}{ccc}
\cos \theta & 0 & -\sin \theta \\
0 & 1 & 0 \\
\sin \theta & 0 & \cos \theta
\end{array}\right] \mathbf{R}^{\prime}(\psi)=\left[\begin{array}{ccc}
\cos \psi & \sin \psi & 0 \\
-\sin \psi & \cos \psi & 0 \\
0 & 0 & 1
\end{array}\right] \\
\mathbf{S}=\mathbf{R}^{\prime}(\phi) \mathbf{R}^{\prime}(\theta) \mathbf{R}^{\prime}(\psi) \\
\mathbf{S}=\left[\begin{array}{ccc}
\sin \psi \sin \theta \cos \psi-\cos \phi \sin \psi & \cos \phi \cos \psi+\sin \phi \sin \theta \sin \psi & \sin \phi \cos \theta \\
\cos \phi \sin \theta \cos \psi+\sin \phi \sin \psi & \sin \theta \cos \phi \sin \psi-\sin \phi \cos \psi & \cos \theta \cos \phi
\end{array}\right]
\end{gathered}
$$

Where $\mathrm{S}$ is the rotation matrix that expresses the orientation of the mobile frame relative to the fixed reference frame.

Additionally the force of gravity $\left(\mathrm{F}_{\mathrm{g}}\right)$ together with the total thrust generated by the propellers $\left(\mathrm{F}_{\mathrm{p}}\right)$ should be equal to the net forces acting on the quadrotor body $\left(\mathrm{F}_{\text {net }}\right)$ :

$$
\mathrm{F}_{g}+\mathrm{F}_{P}=\mathrm{F}_{\text {net }}
$$

We already assumed that quadrotor body being a rigid one the Moment of inertia (I) becomes a diagonal matrix containing only the principal moments of inertia as follows:

$$
\mathbf{I}=\left[\begin{array}{ccc}
I_{11} & 0 & 0 \\
0 & I_{22} & 0 \\
0 & 0 & I_{33}
\end{array}\right]
$$

Substituting the value of I into net moment acting on the quadrotor body $\left(\mathrm{M}_{\text {net }}\right)$, we get: 


$$
\mathrm{M}_{n \varepsilon t}=\left[\begin{array}{ccc}
I_{11} & 0 & 0 \\
0 & I_{22} & 0 \\
0 & 0 & I_{33}
\end{array}\right]\left[\begin{array}{l}
\dot{P} \\
\dot{Q} \\
\dot{R}
\end{array}\right]+\left[\begin{array}{l}
P \\
Q \\
R
\end{array}\right] \times\left[\begin{array}{ccc}
I_{11} & 0 & 0 \\
0 & I_{22} & 0 \\
0 & 0 & I_{33}
\end{array}\right]\left[\begin{array}{l}
P \\
Q \\
R
\end{array}\right]
$$

Consequently, we will have:

$$
\begin{gathered}
\mathrm{M}_{\text {net }}=\left[\begin{array}{c}
I_{11} \dot{P} \\
I_{22} \dot{Q} \\
I_{33} \dot{R}
\end{array}\right]+\left[\begin{array}{c}
\left(I_{33}-I_{22}\right) Q R \\
\left(I_{11}-I_{33}\right) R P \\
\left(I_{22}-I_{11}\right) P Q
\end{array}\right] \\
{\left[\begin{array}{c}
\dot{P} \\
\dot{Q} \\
\dot{R}
\end{array}\right]=\left[\begin{array}{c}
\frac{M_{x}}{I_{11}} \\
\frac{M_{y}}{I_{22}} \\
\frac{M_{z}}{I_{33}}
\end{array}\right]-\left[\begin{array}{c}
\frac{\left(I_{33}-I_{22}\right) Q R}{I_{11}} \\
\frac{\left(I_{11}-I_{33}\right) R P}{I_{22}} \\
\frac{\left(I_{22}-I_{11}\right) P Q}{I_{33}}
\end{array}\right]}
\end{gathered}
$$

(3)

Hence we can find the total thrust generated by the propellers, $F_{p}(2)$ and the net moment acting on the quadrotor body, $\mathrm{M}_{\text {net }}(3)$.

\subsection{Kinematics Of Quadrotor}

The Euler angle sequence consist of three successive rotations viz. angular rate Roll ( $\phi$ ') needs one rotation, Pitch $\left(\Theta^{\prime}\right)$ needs two and Yaw $\left(\psi^{\prime}\right)$ needs three. Hence,

$$
\begin{gathered}
\overrightarrow{\boldsymbol{\omega}}=R(\phi) R(\theta) R(\psi)\left[\begin{array}{l}
0 \\
0 \\
\dot{\psi}
\end{array}\right]+R(\phi) R(\theta)\left[\begin{array}{l}
0 \\
\dot{\theta} \\
0
\end{array}\right]+R(\phi)\left[\begin{array}{l}
\dot{\phi} \\
0 \\
0
\end{array}\right] \\
{\left[\begin{array}{l}
P \\
Q \\
R
\end{array}\right]=\left[\begin{array}{ccc}
1 & 0 & -\sin \theta \\
0 & \cos \phi & \sin \phi \cos \theta \\
0 & -\sin \phi & \cos \theta \cos \phi
\end{array}\right]\left[\begin{array}{l}
\dot{\phi} \\
\dot{\theta} \\
\dot{\psi}
\end{array}\right]}
\end{gathered}
$$

Solving we get,

$$
\begin{gathered}
{\left[\begin{array}{l}
\dot{\phi} \\
\dot{\theta} \\
\dot{\psi}
\end{array}\right]=\mathrm{T}\left[\begin{array}{l}
P \\
Q \\
R
\end{array}\right]} \\
\mathrm{T}=\left[\begin{array}{ccc}
1 & \tan \theta \sin \phi & \tan \theta \cos \phi \\
0 & \cos \phi & -\sin \phi \\
0 & \sin \phi / \cos \theta & \cos \phi / \cos \theta
\end{array}\right]
\end{gathered}
$$

Where $\mathrm{T}$ is the matrix that relates the body-fixed angular velocity vector (w) and the rate of change of the Euler angles. 


\section{Implementation of Quadrotor}

In traditional fixed-pitch quadrotors, stability and flight control are achieved by changing the voltage supplied to each of the four motors which brings a change in the motor revolutions per minutes (RPM) and in turn the thrust generated by each of the propellers. Several detailed descriptions of the modelling of the quadrotors and their dynamics have been published recently. [4] - [7]

We have used ATMEGA 2048 micro-controller and all its codes are written in $\mathrm{C}$ and $\mathrm{C}++$. It is an in house self-developed fight controller, which mates with an I.M.U [INERTIAL MESUREMENT UNIT] together they process data from 17 sensors thus controlling the flight of the quadrotor. When quadrotor is in flight it needs different kinds of measurements to control and keep the quadrotor stable. After the IMU, SONAR and IR readings are acquired, the algorithms reads the last data memorized in the PWM inputs (related to the remote controller). After interpreting and filtering both the sensors and the remote controllers' data the software sets the PWM outputs which through the motor controls the propellers and the flight of the quadrotor. The algorithm can be described as follows:

STEP 1: The readings from the sensors are pre-processed and made into simpler from for the microcontroller to process.

STEP 2: The pre-processed reading are utilized by several $\mathrm{C}++$ functions in the native program of the fight controller to provide the reaction signals to the motor in the form of Pulse Width Modulations (PWM).

STEP 3: The P.W.M signals are utilized by the E.S.C (Electronic Speed Controller), which are attached to each of the four motors, in addition to the independent signals taken from the micro controllers to react to the given situation.

STEP 4: The situation is then performed and the quadrotor is again ready to taking the next command.

\subsection{Quadrotor Architecture:}

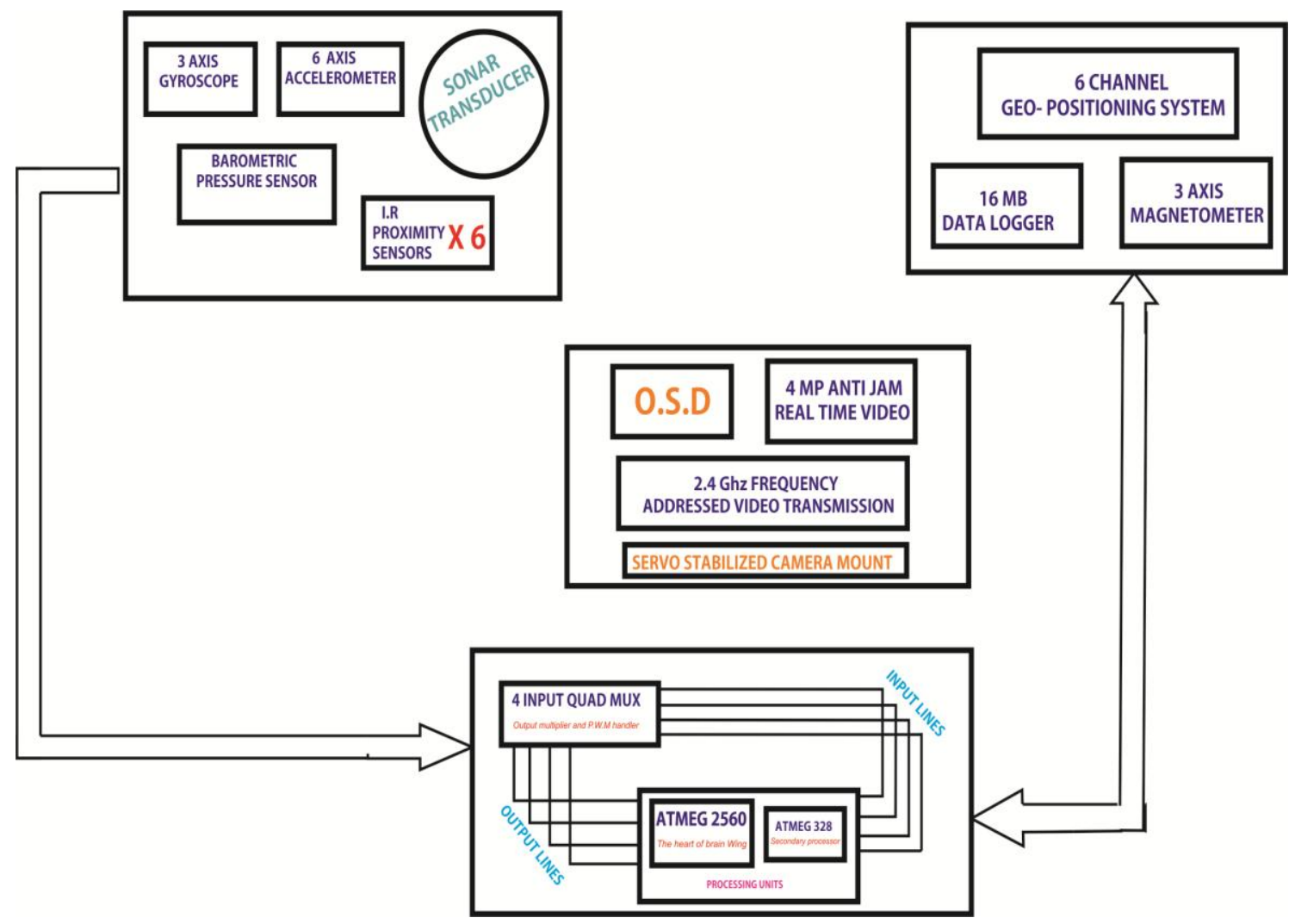

a. Core of the quadrotor is its ATMEGA 2560 microcontroller.

b. This primary ATMEGA 2560 is assisted by a secondary ATMEGA 328 microcontroller, whose primary job is to pre-process reading from different sensors for the primary ATMEGA 2560 microcontroller.

c. The secondary ATMEGA 328 also process the inputs from the radio transmitters, which are generally PWM, and gives it to ATMEGA 2560 in digital I/O format. This makes all the digital ports of the microcontroller to be utilized efficiently.

d. Along with the processors is a multiplexer, CMOS type, which is used to multiplex I/O for the ATMEGA 328 , this makes the reaction faster. 
The different sensors with their parameters used by us are:

a) Gyroscope: Our quadrotor uses a single 3-axis gyroscope to measure the orientation, i.e. to get accurate and fast reaction time by calculating angles in 3 dimensions. It is to be noted that a gyroscope gives only the orientation and not the angle between the fixed reference offset axis and the spinning axis.

b) Accelerometer: Accelerometer measures the rate of the change of orientation, the output of an accelerometer is an unsigned number and the number is scalar, hence it does not show any signs thus we sense only the direction of the rotation.

c) Height Monitoring: In our quadrotor we have used Barometric Pressure (BMP) sensor for measurement of heights above $15 \mathrm{~m}$ and SONAR for maintaining low altitude below $15 \mathrm{~m}$.

1. BMP: Atmosphere has a beautiful features that "Pressure in earth's atmosphere varies with height" and this principle is use in Barometric Pressure (BMP).

2. SONAR: SONAR is the acronym for SOund Navigation And Ranging, which is a sensor capable of measuring a distance using ultrasound waves. The SONAR (combined with an IR module, which is discussed later) is used in this project to estimate the height of the quadrotor from the ground for an altitude below $15 \mathrm{~m}$. Therefore it is mounted on the bottom of the robot, pointing downwards (it has the same direction as the z-axis). Sonar is used instead of BMP at low altitude because pressure in Earth's atmosphere change only after 12 feet.

Some features of the SONAR technology are as follows:

i. Weight of about 12 grams.

ii. Digital data output through $\mathrm{I}_{2} \mathrm{C}$ interface.

iii. Range from 0.03 to $6 \mathrm{~m}$.

iv. Resolution of about $0.01 \mathrm{~m}$

v. Acquisition period of $70 \times{ }^{10-3}$ s. (Frequency of $14 \mathrm{~Hz}$ ).

vi. Beam angle of 55 degrees.

vii. Average power consumption of $50 \times 10^{-3} \mathrm{~W}\left(=10 \times 10^{-3} \mathrm{~A}\right.$ at $\left.5 \mathrm{~V}\right)$.

3. IR Module: IR is the acronym for InfraRed, which identifies a light wave within a certain frequency range.

Some features of the IR Module technology are as follows:

i. Weight of about 7 grams.

ii. Analogue data output.

iii. Range from 0.2 to $1.5 \mathrm{~m}$.

iv. Resolution depending on the distance.

v. Acquisition period of $40 \times 10^{-3} \mathrm{~s}$ (Frequency of $25 \mathrm{~Hz}$ ).

vi. Beam angle less of 5 degrees.

vii. Average power consumption of $150 \times 10^{-3} \mathrm{~W}\left(=30 \times 10^{-3} \mathrm{~A}\right.$ at $\left.5 \mathrm{~V}\right)$.

4. IMU: IMU is the acronym of Inertial Measurement Unit, which is a sensor capable of measuring the orientation (attitude) of the quadrotor through inertial sensors.

Some features of the IMU technology are as follows:

i. Weight of about $100 \mathrm{~g}$.

ii. Digital data output through serial interface.

iii. Acceleration full scale of about $\pm 50 \mathrm{~ms}^{-2}$.

iv. GPS position accuracy of about $2 \mathrm{~m}$.

v. Dynamic accuracy (roll/pitch) of about 1degree.

vi. GPS position update rate of $4 \mathrm{~Hz}$.

vii. Average power consumption of $750 \times 10^{-3} \mathrm{~W}\left(=150 \times 10^{-3} \mathrm{~A}\right.$ at $\left.5 \mathrm{~V}\right)$.

A typical collection of the sensor data can be shown as below:

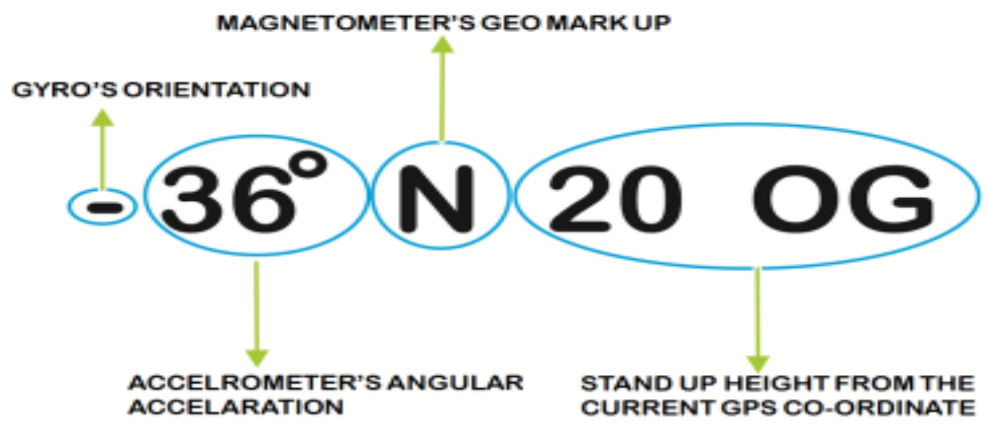




\subsection{Dc Motor:}

The DC Motor is an actuator which converts electrical energy into mechanical energy (and vice versa). It is composed of two interactive electromagnetic circuits. The first one (called rotor) is free to rotate around the second one (called stator) which is fixed instead. Rotors of the quadrotor are these Brush Less DC motors (BLDC, they are known to give the best power to weight ratio). For example, in our quadrotor the brushless motor weighing 100 grams can give around 100 grams of trust per volt and the motor can takes up to $12.3 \mathrm{~V}$, so it can generate a trust anywhere around $1200 \mathrm{grams}(1.2 \mathrm{Kg})$ from each motor which results to a trust of $1200 \mathrm{X} 4=48000(4.8 \mathrm{Kg})$ taking around $3 \mathrm{~kg}$ of self-weight of our quadrotor it can lift pay loads up to $1.8 \mathrm{Kg}$. We have used a motor drive to interface the outputs of the Micro Controller Unit (MCU) to the motors to limit the maximum output current and to provide speed control of the motors.

The Motor-Gear-Propeller system:

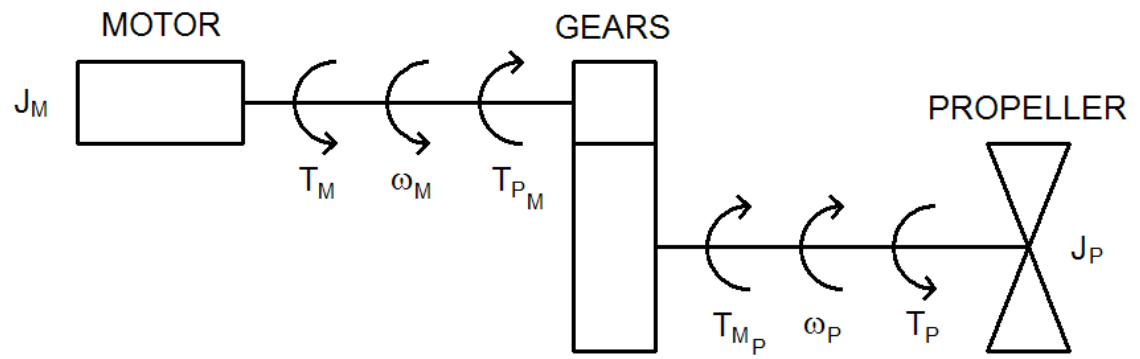

\subsection{The Unshaken Camera And Image Processing:}

Under normal weather and regular airspeed resistance condition, the quadrotor travelled up to $3 \mathrm{~km}$ at stretch, in just 4 minutes. On reaching high altitudes, the quadrotor automatically activates FPV (First Person View) camera mounted on its belly. Quadrotor uses ZigBee protocols and stealth algorithms (embedded in to its main $\mathrm{C}++$ functions) making the communication between the ground station and the quadrotor invisible to nonaddressed radio transceivers, this makes the channel highly impossible to get jammed by external radiations and thus creating highly secured communication channel between the ground station and the quadrotor. The output from the accelerometers and gyroscopes are also used to provide stability to the camera.

The output of the MCU is connected to a high precision servo which controls the movement of the camera. The programming is done in such a manner that camera inputs the offset position from the user selected point of view, this point is selected such that it behaves the offset to quadrotor from the time instance and the program always maintain this offset with help of the inputs from the gyroscope and accelerometer and these inputs are maintained using the actuators (servos). This makes the implementation of image processing algorithms an easy task since the image stability is achieved in the hardware itself. Once the stability of the FPV camera is achieved the captured real time videos are sent using a $\mathrm{RF}$ wireless camera at $2.4 \mathrm{GHz}$. These videos are then converted to snapshots which are then processed using MATLAB-Image Processing Toolbox to count the number of persons in that specific captured image. We performed a trial test to see if our code works and we found satisfactory results where we counted number of people in a crowd, which came out to be 5239. A snapshot of the same is shown below.
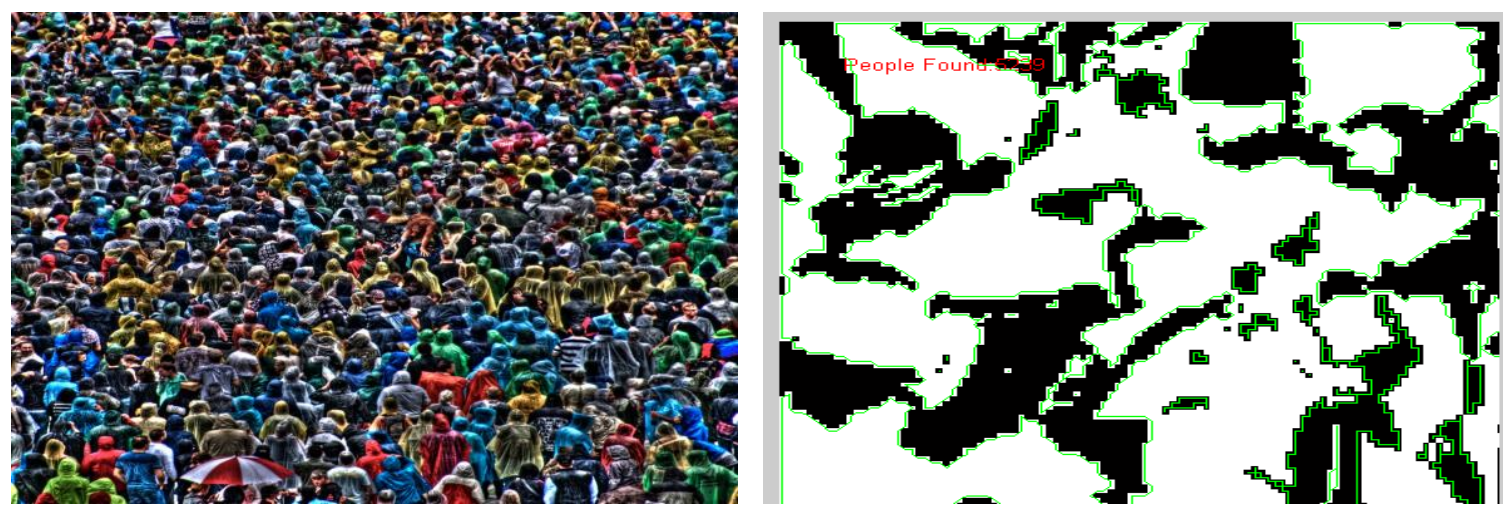

\section{Conclusion}

In this paper we discuss an Unmanned Aerial Vehicle (UAV), called quadrotor which has vertical takeoff and landing (VTOL) capabilities which gives it higher manoeuvrability and hovering capabilities. This paper presents a complete system solution to the development of a fully functional quadrotor capable of hovering 
starting from dynamic modelling of the quadrotor, kinematic modelling of the quadrotor, implementation of the wireless camera for continuous transmission of video and finally the image processing performed on the captured image. The particular interest of the research community in the quadrotor design can be linked to two main advantages over the conventional aircrafts, such as helicopters. First, quadrotors do not require complex mechanical control linkages for rotor actuation, relying instead on fixed pitch rotors and using variation in motor speed for vehicle control. This simplifies both the design and maintenance of the vehicle. Second, the use of four rotors ensures that individual rotors are smaller in diameter than the equivalent main rotor on a helicopter, relative to the airframe size.

The one thing we have done here is that hardware on our quadrotor is modular and shielded, i.e. one module mates on the other without any wires in between them and this reduces the dead weight of the quadrotor. Also the modular design makes part replacement very easy and affordable. Other noticeable advantage we could do was to operate the quadrotor at very low voltages by making it work on the reverse current produced by the motors.Due to these abovementioned specific capabilities, Unmanned Aerial Vehicles (UAVs) have found potential applications, both as individual vehicles and in multiple vehicle teams, in military purposes like surveillance, border patrolling, mine detection, crowd control, aerial delivery of payload, civilian purposes like disaster management during floods, earthquakes, fire, commercial missions like aerial photography, television and cinema shootings, and research and development program which need flying vehicles to perform various experiments. The camera mounted on the belly of the quadrotor can be used to determine the position of a particular object, tracking of mobile targets and for environment mapping.

\section{Acknowledgment}

We would like to acknowledge the guidance of our project guide and mentor Mrs Pragati Gupta (Assistant Professor, Department of Electrical Engineering, V. J. T. I, Mumbai)

Websites:

\section{Refferences}

[1]. J. G. Leishman. The Breguet - Richet Quad Rotor Helicopter of 1907,

[2]. http://www.glue.umd.edu/_leishman/Aero, accessed Jan. 2009.

[3]. $\quad$ PRNewswire (Feb 1, 2010). Teal Group Predicts Worldwide UAV Market

[4]. Will Total Over $\$ 80$ Billion in its Just Released UAV Market Profile and

[5]. Forecast. PRNewswire - January 2011 from

[6]. http://www.prnewswire.com/news-releases/teal-group-predicts-worldwideuav-market-will-total-over-80-billion-in-its-just-released2010-uav-marketprofile-and-forecast-83233947.html

\section{Books:}

[7]. J. G. Leishman. Principles of helicopter aerodynamics. (Cambridge University Pr, 2006).

\section{Journal Papers:}

[8]. M. Y. Amir and V. Abbass. Modelling of Quadrotor Helicopter Dynamics. In International Conference on Smart Manufacturing Application (ICSMA 2008), pages 100 (105, April 2008).

[9]. B. Erginer and E. Altug. Modelling and PD Control of a Quadrotor VTOL Vehicle. In IEEE Intelligent Vehicles Symposium, pages 894 \{899, June 2007).

[10]. M. Alpen, K. Frick, and J. Horn. Nonlinear Modelling and Position Control of an Industrial Quadrotor with On-Board Attitude Control. In IEEE International Conference on Control and Automation, pages 2329 \{2334, December. 2009).

[11]. J. Kim, M. S. Kang, and S. Park. Accurate Modelling and Robust Hovering Control for a Quadrotor VTOL aircraft. Journal of Intelligent and Robotic Systems, 57(1):9(26, 2010). 